\title{
Exister au risque de disparaître. Récits sur la mort pendant la traversée vers l'Europe
}

Existing at Risk of Disappearing. Narratives on Death during Border Crossing

Existir arriesgando desaparece. Relatos sobre la muerte durante la travesía

hacia Europa

\section{Carolina Kobelinsky}

\section{(2) OpenEdition}

Journals

Édition électronique

URL : https://journals.openedition.org/remi/8745

DOI : 10.4000/remi.8745

ISSN : $1777-5418$

Éditeur

Université de Poitiers

Édition imprimée

Date de publication : 1 septembre 2017

Pagination : 115-131

ISBN : 979-10-90426-59-7

ISSN : 0765-0752

Référence électronique

Carolina Kobelinsky, « Exister au risque de disparaître. Récits sur la mort pendant la traversée vers

l'Europe », Revue européenne des migrations internationales [En ligne], vol. 33 - n² et 3 | 2017, mis en ligne le 01 septembre 2019, consulté le 14 avril 2022. URL : http://journals.openedition.org/remi/8745 ; DOI : https://doi.org/10.4000/remi.8745 


\title{
Exister au risque de disparaître. Récits sur la mort pendant la traversée vers l'Europe
}

\section{Carolina Kobelinsky ${ }^{1}$}

\author{
À Paris, commentant la nouvelle, qu'on entendait à la radio, du naufrage en \\ Méditerranée d'une embarcation remplie de migrant·e.s, Ibrahim Sagara, qui venait \\ d'emprunter les mêmes routes quelques mois auparavant, commente d'un ton laconique : \\ "Aux frontières, c'est mourir ou disparaître... ou bossa. ${ }^{2}$ "
}

À Melilla, enclave espagnole bordant la mer Méditerranée, les migrant·e.s racontent de nombreuses histoires sur la mort pendant la traversée vers I'Europe. Depuis plusieurs décennies, les morts des hommes, femmes et enfants aux frontières de l'Europe sont nombreuses. La découverte de corps noyés, déshydratés, asphyxiés, intoxiqués sur les côtes de la Méditerranée, de l'Égée, de l'Atlantique, tout comme aux frontières terrestres de I'Union, est devenue une réalité quotidienne ${ }^{3}$. À Lampedusa comme à Lesbos ou àTenerife, des tombes et des cases de columbarium sans nom matérialisent la violence dont sont actuellement porteuses les frontières européennes. Sans être le produit d'États totalitaires ou dictatoriaux, la dureté du traitement migratoire - les politiques restrictives, les techniques sophistiquées de contrôle, les discours criminalisant les étrangers, les morts invisibles dans les statistiques officielles ${ }^{4}$ - crée un espace qui normalise la mort des migrant.e.s au cours de leur voyage. À cette violence structurelle (Weber et Pickering, 2011) et ultime, qu'Albahari (2015) nomme des "crimes de paix", se greffent d'autres formes de violence (physique, symbolique) exercées tout le long de la frontière extérieure de l'Union européenne telles que les refoulements musclés, les souffrances infligées aux corps, la

\footnotetext{
1 Anthropologue, chargée de recherche au CNRS, Laboratoire d'ethnologie et de sociologie comparative (LESC), Maison Ethnologie \& Archéologie René-Ginouvès, 21 allée de I'Université, 92023 Nanterre ; carolina.kobelinsky@cnrs.fr

2 Mot prononcé pour signifier la réussite dans le passage de la frontière. On entend souvent les migrant.e.s crier "bossa, bossa " lorsqu'ils essaient de sauter les clôtures. Selon certains interlocuteurs, cela voudrait dire victoire et viendrait d'une déformation d'un terme wolof.

3 Voir la cartographie des morts produite par le réseau Migreurop (2012) ainsi que le numéro 109 de Plein droit consacré aux "Homicides aux frontières " (2016).

4 II n'existe à I'heure actuelle aucun comptage officiel au niveau européen de ces morts de la migration. Pour consulter une recension élaborée par un consortium de journalistes à partir des chiffres des morts avancés par différentes ONG, se référer à la base de données The Migrant Files. Cf. http://www.themigrantsfiles.com/ (consulté le 18/08/2016).
} 
non-assistance à personnes en détresse (Heller et Pezzani, 2014) ${ }^{5}$ et l'absence de protocole commun d'identification et de prise en charge des morts de la migration (Kobelinsky, 2015) ${ }^{6}$.

Contrairement aux frontières maritimes, peu de morts sont comptabilisées et peu de corps sont retrouvés à la barrière de Melilla. La mort est pourtant un spectre qui accompagne l'expérience de toutes les personnes que j'ai rencontrées dans l'enclave et ses alentours. Cette omniprésence spectrale constitue elle aussi une forme de violence à la frontière ; elle est un effet de la frontiérisation ou bordering européenne (Cuttitta, 2015a ; Ritaine, 2015), qui s'infiltre tout autant dans les récits des expériences vécues que dans les rumeurs qui circulent des deux côtés de la frontière. Ces rumeurs parlent de migrant.e-s disparu.e-s à jamais. Je m'intéresserai ici à la forme et au contenu de ces discours, en explorant ce qu'ils véhiculent et ce qu'ils disent de la migration aujourd'hui. Ces histoires constituent une entrée ethnographique permettant d'explorer la réalité de la migration contemporaine et - tel que le suggère Melly (2011 : 373) dans son étude des histoires des hommes absents au Sénégal - d'examiner les anxiétés collectives de la migration. Les récits dont il est question dans l'article ont été recueillis au cours d'un travail de terrain conduit entre 2014 et 2016 notamment auprès de migrantee.s, mais également d'acteurs associatifs, se trouvant dans la province marocaine de Nador et dans l'enclave espagnole de Melilla ${ }^{7}$. La plupart du matériau mobilisé ici provient de conversations informelles auprès $\mathrm{d}^{\prime}$ hommes - entre dix-sept et trente-cinq ans - originaires d'Afrique de l'Ouest ${ }^{8}$. J'ai parallèlement conduit quelques entretiens qui ont pu être enregistrés. La principale langue des échanges a été le français, qui n'est pas la langue maternelle de mes interlocuteurs, mais qui est celle à travers laquelle ces migrant·e.s échangent entre eux. Cette langue commune est agrémentée de termes issus d'origines diverses qui nomment des objets, des personnes et des actions de leur vie de migrant·es. Se crée ainsi un vocabulaire partagé de la frontière qui emprunte des termes ou des formulations au wolof, au peul, au nouchi (I'argot ivoirien), à l'espagnol, à l'arabe. Quelles que soient les origines, pour toutes les

5 Voir également à cet égard le rapport de Forensic Oceanography élaboré en collaboration avec Watch the Med. Cf. https://deathbyrescue.org/ (consulté le 29/05/2017).

6 C'est ce que De León (2015) nomme dans son étude deș migrant·e·s décédé·e·s en tentant de traverser le désert qui sépare le Mexique des États-Unis, une forme de " nécro-violence ", c'est-à-dire une violence performée et produite à travers le mauvais traitement des cadavres.

7 Cette enquête de terrain a été effectuée dans le cadre d'une recherche plus large, en cours depuis 2013, sur la prise en charge des morts aux frontières sud de l'Europe où je $m^{\prime}$ intéresse particulièrement aux trajectoires spatiales des corps et aux pratiques et motivations des acteurs engagés, de façon officielle ou informelle, dans la gestion de ces morts de la migration (identification des corps, enterrement, rapatriement, cérémonies d'hommage, etc.) Ce travail est poursuivi dans le cadre du programme MECMI (financement ANR et FRSCQ).

8 II est plus difficile d'enquêter auprès des migrantes à Melilla, car elles restent la plupart du temps dans le CETI (Centro de Estancia Temporal para Inmigrantes), centre de séjour temporaire où elles sont hébergées. Si elles ont le droit de sortir, les personnes extérieures n'y ont pas accès. L'absence de légitimité d'une femme, surtout si elle est seule et loin de chez elle, à être dans l'espace public explique probablement que ces dernières préfèrent rester dans I'enceinte du CETI. Cet article se concentre donc sur les discours émis par des interlocuteurs hommes. Je laisserai aussi de côté ceux des jeunes garçons d'origine marocaine présents à Melilla, pour qui la mort est également omniprésente, mais dont les mises en mots doivent être examinées à la lumière de leurs yeux d'enfants, et qui plus est, d'enfants isolés habitant dans la rue. 
personnes rencontrées, bossa signifie la réussite de la traversée des frontières ; Guardia nomme un agent des forces de l'ordre espagnoles ${ }^{9}$.

\section{Le dispositif " anti immigrés " à Melilla}

Avec l'entrée de I'Espagne dans I'Union européenne et la promulgation de la première loi espagnole en matière d'immigration en 1985, les enclaves de Ceuta et Melilla - toutes deux situées à l'intérieur du continent africain - se transforment. Une grande partie de leur population, d'origine marocaine et de confession musulmane, ne possédait pas de citoyenneté ni de résidence espagnole. La nouvelle loi prévoyait un processus de régularisation administrative, mais les réticences à sa mise en œuvre ont conduit à de nombreuses mobilisations pour revendiquer l'accès à la nationalité. Finalement, le gouvernement a approuvé en 1986 la naturalisation des personnes résidant dans les deux enclaves ; processus qui s'est déroulé entre 1986 et 1990. Peu de temps après est introduite l'obligation de visa pour entrer dans le pays pour les ressortissants marocains ${ }^{10}$, tunisiens et algériens. Durant cette même période, la presse nationale - les quotidiens El País, El Mundo entre autres - commence à faire écho de l'arrivée sur les côtes andalouses des embarcations de pêche, construites en bois, connues sous le terme de pateras, qui traversent le détroit de Gibraltar en transportant des personnes cherchant à entrer en Espagne de façon irrégulière (Gabrielli, 2011 : 137-138). C'est aussi vers la fin des années 1980 et le début de la décennie suivante que l'on commence à compter les premiers morts aux frontières de l'Europe ${ }^{11}$. Les images de migrants débarquant sur les côtes renvoient à un imaginaire de l'invasion et de l'envahissement sur lequel s'appuie la justification de mettre en place une technologie de contrôle et de surveillance tout le long des frontières espagnoles. L'objectif est ici le même que partout aux frontières extérieures de I'Union européenne : empêcher les arrivées. À Melilla cela se traduit par l'érection de barrières.

Lieu devenu iconique d'une Europe qui se fortifie derrière des murs, Melilla est actuellement considérée comme un exemple de bonne gestion des frontières $^{12}$ et un modèle suivi pour la mise en place de contrôles frontaliers. La construction d'une première barrière de presque douze kilomètres de long - qui couvre la totalité de la frontière terrestre - a débuté dans cette ville autonome en 1998. Une deuxième barrière de trois mètres de hauteur a été construite par

9 Ce vocabulaire ne fera pas l'objet de cette étude, mais j'expliciterai le sens de certains termes utilisés par mes interlocuteurs au fil des pages.

10 Seuls les Marocains résidant dans les provinces de Nador et Tétouan sont exemptés de visa pour se rendre dans les enclaves voisines afin de garantir le commerce transfrontalier.

11 Le blog du journaliste Gabriele del Grande, le premier à recenser les migrant.e.s mort-e.s en tentant de franchir les frontières européennes, à partir des articles de presse, inscrit la date du 1er novembre 1988 comme celle des premiers décès, survenus à la suite du naufrage d'une petite embarcation au large de Cadix, faisant dix morts et neuf personnes disparues. Cf. http://fortresseurope.blogspot.fr/p/la-strage.html (consulté le 18/08/2016).

12 Le journal El País a consacré sa une du samedi 30 avril 2016 ainsi que de nombreux articles à examiner le " succès politique " du traitement migratoire à Melilla ainsi que son " échec moral ". Cf. http://elpais.com/hemeroteca/elpais/portadas/2016/04/30/ et http:// politica.elpais.com/politica/2016/04/28/actualidad/1461840514_222480.html (consultés le 18/8/2016). 
la suite en parallèle à la première. En 2005, le gouvernement a décidé, après plusieurs tentatives de passage de migrant.e.s, de rehausser les grillages à six mètres et de construire un cordage tridimensionnel, connu comme troisième valla (barrière), dont le but est d'empêcher le passage entre la première et la deuxième barrière (APDHA, 2014). Le Maroc a récemment creusé à certains endroits stratégiques un fossé juste devant la barrière, entre celle-ci et la route (APDHA, 2016 : 53). Des barbelés longent les clôtures et un réseau de câbles souterrains est relié à des capteurs électroniques de bruit et de mouvement. La surveillance est complétée par un système de caméras vidéo de vision nocturne. Des postes de surveillance sont répartis le long des grillages et les routes entre les barrières permettent le passage constant de véhicules de la Guardia Civil ${ }^{13}$ qui effectue des rondes. Suite à des accords avec le Maroc, la surveillance a également été renforcée de l'autre côté des barrières par des effectifs de la gendarmerie royale marocaine ${ }^{14}$. Si les clôtures " anti immigrés", comme les désignait un agent de la Guardia Civil lors d'une discussion informelle, n'arrêtent pas la migration, elles rendent le passage de cette frontière extrêmement difficile et dangereux.

Depuis 2015, les arrivées par les clôtures de Melilla ont diminué de façon considérable, les tentatives sont plus rares et les réussites plus extraordinaires. Selon le gouvernement espagnol, autour de 4000 personnes ont tenté de sauter les clôtures de Melilla en 2016, mais seules 332 ont réussi à les franchir, alors qu'au cours de l'année 2015, 18000 personnes ont tenté de traverser les grillages et 2229 y sont effectivement parvenus ${ }^{15}$. Plusieurs éléments permettent de comprendre cette nouvelle réalité : les derniers renforcements des vallas rendent l'exploit encore plus difficile ; les fréquentes descentes des forces de l'ordre marocaines dans les campements des migrant·ess sur le Gourougou montagne qui se trouve à quelques kilomètres seulement de Melilla et où s'organise la vie des migrant·e.s et l'entraînement physique pour escalader les barrières - éparpillent et désorganisent quelque peu les groupes ${ }^{16}$; les " devoluciones en caliente ", expulsions immédiates et collectives des personnes qui viennent juste de toucher le sol espagnol ${ }^{17}$, fréquemment pratiquées après un

13 Force de police à statut militaire, comparable à certains égards à la gendarmerie nationale française, la Guardia Civil exerce des missions multiples : sécurité publique, police judiciaire, missions à caractère militaire et relevant du domaine fiscal.

14 Sur I' " industrie " qui se développe autour de la surveillance des frontières, voir les travaux de Rodier (2012) et Andersson (2014).

15 Cf. http://www.eldiario.es/politica/Solo-inmigrantes-lograron-saltarMelilla_0_632737111.html (consulté le 29/05/2017).

16 Les tentatives de sauter les grillages se font collectivement, jamais de façon individuelle et non organisée. Sur certains de ces éléments permettant de comprendre le tarissement actuel de cette stratégie de passage, cf. http://www.eldiario.es/desalambre/ saltos-valla-Melilla_0_413659426.html (consulté le 18/08/2016).

17 Largement critiquée pour être contraire à la Convention européenne des droits de I'homme, la " devolución en caliente " était couramment employée à Melilla. En avril 2015, la loi espagnole introduit la figure du "rechazo en frontera " (expulsion à la frontière), légalisant de ce fait une pratique déjà en place. Depuis, une équipe de prestigieux juristes a élaboré un rapport argumentant l'illégalité de cette légalisation au regard de la Constitution espagnole et les accords internationaux en matière de droits de I'homme. Le rapport est consultable en ligne. Cf. http://www.parlament.cat/document/ intrade/59171 
salto (saut), ont pu avoir un effet dissuasif ${ }^{18}$.

Ainsi, si presque la totalité des personnes que j'ai rencontrées à Melilla au cours de mes deux séjours en 2014 étaient arrivées par la valla, la plupart de celles que j'ai connues en 2016 avaient emprunté d'autres voies pour entrer sur le territoire espagnol après une ou plusieurs tentatives infructueuses d'escalader les grillages. Les stratégies actuellement suivies sont la nage le long de la côte, I'usage de petites embarcations de fortune, construites à partir de matériaux trouvés sur place, ainsi que de canots gonflables, l'infiltration par les conduits d'eau, la dissimulation dans des voitures et des camions où il est possible de se cacher à plusieurs pour traverser l'un des quatre postes frontières de la ville contre une somme considérable d'argent remise au passeur, l'achat de faux papiers marocains permettant de traverser les contrôles ${ }^{19}$.

Une fois à Melilla, les migrant-e.s sont conduit.e.s au Centre de séjour temporaire pour immigrés, appelé le CETI (Centro de Estancia Temporal para Inmigrantes), ouvert en 1999, en même temps que s'érigeaient les clôtures. Le CETI se trouve à proximité du poste frontière de Farhana, en périphérie de la ville et - ironie du paysage local - juste à côté d'un terrain de golf où des habitants fortunés de la ville viennent se divertir. À la différence des centres de rétention, nommés en Espagne centres d'internement d'étrangers ou CIEs, qui dépendent du ministère de I'Intérieur, le CETI dépend du ministère de I'Emploi et de la Sécurité sociale ${ }^{20}$. Il s'agit d'un établissement conçu comme un " dispositif de premier accueil prévisionnel et destiné à offrir des services et des prestations sociales basiques au collectif d'immigrés et demandeurs d'asile qui arrivent [...] pendant le déroulement des démarches d'identification et le bilan de santé préalables à toute décision sur la suite la plus adéquate en fonction de leur situation administrative en Espagne ${ }^{21}$. Les personnes prises en charge au CETI sont ainsi en attente d'être envoyées vers la Péninsule ibérique, appelée la salida (sortie) dans le jargon des gestionnaires du centre et des migrant.e.s. Une fois de l'autre côté de la Méditerranée, elles peuvent être enfermées dans un centre d'internement afin d'organiser leur expulsion vers leur pays d'origine ou bien être accueillies dans différents établissements associatifs qui leur apportent un suivi sanitaire, social et juridique, en cas de demande d'asile, pour une durée déterminée. S'articulent ici assistance et contrôle, compassion et répression, humanitarisme et sécurisation, les dimensions complémentaires du régime européen des frontières contemporaines (Agier, 2008 ; Andersson, 2014 ; Cuttitta, 2015b ; Fassin, 2005 ; Pallister-Wilkins, 2015).

\footnotetext{
18 Or, celui-ci est impossible à mesurer, tout comme il n'est pour l'heure pas possible de chiffrer la capacité dissuasive de l'arsenal sécuritaire déployé tout le long de la frontière européenne.

19 Tel que m'expliquait un jeune Congolais, cette dernière stratégie peut être considérée " seulement si t'es clair ", sous-entendant que la plupart des Subsahariens ne peuvent pas faire semblant d'être Marocains en raison de leur couleur de peau. C'est d'ailleurs ce que note I'Asociación Pro Derechos Humanos de Andalucía dans son dernier rapport (APDHA, 2016 : 29-30).
}

20 II existe seulement deux CETIs en Espagne, I'un à Melilla, I'autre à Ceuta. Tous les deux se trouvent sous la même tutelle et possèdent les mêmes compétences. 
Le CETI de Melilla a une capacité de 472 places, mais il est souvent saturé. Au printemps 2014, plus de 1900 personnes y attendaient depuis plusieurs mois, voire des années ${ }^{22}$ leur salida vers la Péninsule, espérant qu'elle n'aboutirait pas à une expulsion vers leurs pays d'origine. Les portes du CETI - contrairement à celles des $\mathrm{CIEs}$ de la Péninsule - restent ouvertes la journée. Les résidents peuvent ainsi entrer et sortir de l'établissement ${ }^{23}$. Enfermés dans I'enclave et en situation irrégulière, ils n'ont pas le droit de travailler. Le rythme quotidien est ainsi marqué, pour la plupart, par les horaires des repas à la cantine, éventuellement par les moments de prière ou des réunions d'information organisées par des associations. Certains essaient de gagner un peu d'argent, en proposant, un seau et une éponge à la main, de laver les voitures garées dans le centreville ou en poussant les caddies des courses depuis la porte des supermarchés jusqu'aux voitures.

\section{Frôler la mort, risquer la disparition}

Après avoir été envoyé à Agadir, à 1100 kilomètres de Melilla, à la suite d'une tentative infructueuse de sauter les clôtures et sans argent pour payer un passeur qui pourrait I'amener en Europe, Ibrahim Sagara, jeune homme originaire du Mali, décide de tenter sa chance à la nage. II sait nager depuis l'enfance puisqu'il a grandi tout près d'un fleuve où il avait l'habitude de s'amuser avec les autres enfants du village. II commence alors à s'entraîner tous les jours. II fait la manche - ce qu'il appelle, comme la plupart de mes interlocuteurs, "taper Salam " - et s'achète d'abord une combinaison, plus tard une petite planche de plage pour enfants en polystyrène. Quand il se sent prêt et qu'il a l'argent nécessaire au voyage, il prend un bus pour Fès. Sa planche, qu'il a placée dans le compartiment à bagages au-dessus de sa tête, est cassée par le poids du grand sac qu'une dame a posé par-dessus. Seul Noir dans le bus, il m'explique qu'il n'a pas eu le courage de lui faire la remarque. À Fès, avec un peu d'argent " du Salam ", il s'achète du scotch pour réparer la planche et un billet pour Nador. II quitte la ville sans dire aux compatriotes avec qui il venait de passer quelques jours qu'il a l'intention de rejoindre Melilla ce jour même à la nage. II garde le secret de peur d'entendre ses camarades tenter de le décourager. Arrivé à $19 \mathrm{~h}$ sur une plage de Nador, il enlève ses habits, ses chaussures et les jette à la mer. Pour ne pas alerter les policiers marocains, il ne veut pas laisser de traces sur la plage. Il regarde les lumières de Melilla, à plus de seize kilomètres et se dit : " J'y serai dans la nuit, je suis sûr que je peux le faire ". II commence à nager, s'éloigne de la côte pour ne pas être repéré et se repose sur la planche lorsqu'il est trop fatigué. II raconte que durant la traversée il a perdu la notion du temps, qu'il se guidait par les lumières au loin. Ibrahim arrive au port de Melilla - où il est découvert par la Guardia Civil - après avoir nagé plus de quatorze heures. Lorsqu'il me raconte en détail son expérience, il me dit qu'il n'a pas eu peur de mourir sur le coup, mais rétrospectivement :

22 À titre d'illustration, de nombreux ressortissants du Bangladesh ont passé trois, quatre et jusqu'à cinq ans à Melilla avant d'obtenir leur salida et d'être directement envoyés au centre de rétention de Barcelone. Cf. http://periodismohumano.com/ migracion/camino-de-la-expulsion-5-anos-despues.html (consulté le 18/08/2016).

23 Sur I'organisation spatiale du CETI et les expériences des migrant·e.s pendant I'attente, consulter I'article de Bondanini (2014). 
" J'étais pris dans l'activité [de nager], je n'ai pas du tout, du tout pensé à ça lorsque je nageais, j'étais concentré, concentré. Je n'ai même pas vu les poissons autour! On voit pas grand-chose la nuit. J'étais concentré dans les lumières et les courants, je ne voulais pas dévier [...] et j'avais au fond de moi mon rêve [arriver en Europe et devenir musicien]. Quelques jours plus tard, je me suis réveillé et j'avais peur. [II crie, en cherchant un effet comique] Ah ! J'étais en sueur ! J'ai eu peur là, j'aurais pu mourir et personne n'aurait rien su, on ne m'aurait jamais retrouvé, j'aurais disparu. "

(Conversation, 20/04/2016)

Les récits sur les tentatives - heureuses ou frustrées - de franchir les frontières comportent souvent une dimension liée à la proximité de la mort et sont parfois racontées en cherchant à provoquer le rire ou en soulignant le pittoresque. $C^{\prime}$ est ce que fait Ibrahim Sagara, qui ne cherche pas à magnifier son exploit - à l'inverse de ce que font par ailleurs ses voisins de chambre au CETI qui lui rappellent chaque jour son arrivée héroïque -, mais plutôt à me faire sourire. Raconter son expérience sous la forme d'une aventure lui permet comme à tous ceux que j'ai entendus faire le récit de la traversée des frontières de cette manière-là - de mettre à distance ce qu'il craint, d'apprivoiser le risque encouru, en le transformant en matière à rire ${ }^{24}$. Dans son discours, Ibrahim Sagara met en mots la peur de la mort et de la disparition. Il fait d'ailleurs une distinction entre la possibilité de mourir et celle de disparaître, qui est plus clairement mise en évidence lors d'une conversation ultérieure :

"On sait qu'on peut mourir, on le comprend vite, mais tu continues parce qu'il y a quelque chose qui te pousse [...]. Je pense parfois que la vie n'est rien, c'est fragile, tu peux mourir comme ça [il fait un geste rapide de sa main], tu meurs et c'est fini, ta famille pleure pour toi, tes amis [...]. [Mais] si personne le sait, ta mère ne l'apprend pas, tu disparais dans la nature, tout s'arrête, mais personne ne sait que c'est fini, fini pour toi, tout est suspendu. " (Conversation, 04/05/2016)

La mort, distinguée ici de la disparition, apparaît comme la fin normale de la vie. Le mort est pris en charge par la communauté, s'inscrit dans des rituels déjà connus (Bloch et Parry, 1982). II occupe une place définie dans la société. En cela il continue d'exister. Le disparu, à l'inverse, ne subsiste que comme une énigme. Ne connaissant pas son sort, la famille ne peut pas le " pleurer ". À la fois absence physique et sociale, la disparition constitue un effacement imperceptible qui " suspend " la personne disparue dans un espace/temps particulier. Cette distinction apparaît également lors d'une conversation avec Cellou Diallo, Peul de dix-huit ans, originaire de Conakry (Guinée), avec qui j'avais I'habitude de discuter assis sur un banc du parc Hernández - qui se trouve au cœur du centre-ville de Melilla et que mes interlocuteurs nomment "le jardin ". Au cours d'une discussion anodine, il me raconte à la première personne l'expérience d'avoir assisté à la mort de pairs, d'avoir dû les laisser derrière, sur le chemin vers l'Europe :

24 Dans la lignée des travaux de Freud (1992), des nombreuses études ont analysé I'humour comme une manière de résister, au moins symboliquement, au danger que I'on ne peut éviter (Gay-Para, 1986 ; Matard-Bonucci, 1998 ; Moussaoui, 2013) ou que I'on redoute le plus (Clastres, 1974). 
"C'était dur le désert, j'ai vu des grand-frères mourir, c'était vraiment dur, laisser

un grand derrière toi, le voir mourir et continuer. Tu marches, $t^{\prime}$ avances, mais t'as l'impression que tu n'avances pas, t'en peux plus et tu penses à ceux qui viennent de mourir et qui sont derrière. En Algérie, j'ai regretté d'être parti. Je l'ai regretté plusieurs

fois pendant le voyage, mais dans le désert [silence] quand le grand est décédé juste à côté de moi. Là j'ai regretté, mais c'était trop tard, je ne pouvais pas retourner et puis le souvenir de ce que j'ai vu, des grand-frères, je ne l'oublierai pas, c'était vraiment dur, j'aurais pu mourir aussi comme le grand, comme les autres. J'aurais disparu au milieu du sable et ma mère n'aurait rien appris, cela n'aurait pas été connu de ma famille, j'aurais disparu complètement. " (Conversation, 24/05/2016)

Le régime contemporain des frontières - avec sa technologie de plus en plus sophistiquée - pousse Cellou Diallo et bien d'autres migrant.e.s vers des zones d'exposition aux "faits de la nature ", transformant ici le désert - et dans le récit précédent la mer - en des scènes de mort (Schindel, 2016). Pour Cellou Diallo, disparaître dans le désert c'est y rester physiquement indéfiniment, jamais son corps ne retrouvera les siens, ce qui constitue une perspective effrayante. Cependant, pour la plupart de mes interlocuteurs, le sort de leur dépouille leur paraissait moins important que l'annonce du décès à la famille. Beaucoup acceptaient l'idée que leur corps ne soit jamais enterré, aucun ne supportait la perspective de laisser leurs proches dans l'ignorance de leur mort. Informer la famille c'est exister comme mort. Être seulement disparu c'est risquer de rester perpétuellement dans une place indéterminée au sein de la société.

Ce que Cellou Diallo nomme une disparition " complète " apparaît dans les termes de Mamadou Condé comme une disparition " totale ". Ce Guinéen de vingt-six ans me raconte sous la forme d'un inventaire détaillé tous les morts qu'il a vus au cours de sa traversée et commente sur la fin de sa diatribe le sort des morts " que personne n'a vus " :

" J'ai vu des morts depuis que j'ai quitté la Guinée, j'ai vu avec mes yeux. Une femme n'a pas survécu les conditions dans le désert, c'était dur pour tous, trop pour elle. Au Maroc j'ai vu un jeune Malien mourir aux mains de la police, mais il n'avait rien fait de mal, ils l'ont tabassé à mort. Et dans le bateau à cause du moteur cassé deux personnes sont décédées, une femme est décédée intoxiquée [par inhalation de produits toxiques] d'abord et après un homme est décédé aussi [...] et dans le Gourougou c'est des mortsvivants que j'ai vus [...] et ceux que j'ai vus, qu'on a vus et dont on te parle ce n'est $q u$ 'une petite partie, tous les frères qu'on a pas vus, qui sont totalement disparus, pour toujours, dont personne n'aura plus de nouvelles. " (Conversation, 06/11/2014)

Le bilan dressé par Mamadou Condé détaille autant les morts que les causes de leur décès. Il soulignera à plusieurs reprises au cours de la conversation que le fait d'avoir été là, d'avoir assisté à ces morts a été une "épreuve ". Quand il parle de la vie au Gourougou, il emploie le terme " morts-vivants " - terme que j'ai souvent entendu pour faire référence à la vie qui se déroule dans les campements où des centaines (voire des milliers selon les périodes) de migrant.e.s attendent de réussir le passage des frontières. Or, il intègre ces " mortsvivants " dans l'inventaire des morts de son parcours. Par contraste, ces récits sur la " presque " mort parlent de cette " vraie " vie qu'ils espèrent derrière les barrières. Cette mise en mots - plus que l'expérience elle-même - reprend 
la forme du rite de passage ${ }^{25}$. Elle souligne le changement de statut auquel est censée aboutir la migration et permet ainsi de donner du sens à la souffrance, notamment celle du corps, devenue la condition nécessaire au surgissement d'une nouvelle identité sociale dont ils sont à la recherche. Comme Cellou Diallo et Ibrahim Sagara, Mamadou Condé associe la disparition à l'impossibilité d'annoncer le décès aux proches. Mais il y ajoute une dimension supplémentaire, qui apparaît en filigrane dans son récit à travers les répétitions du terme "voir ", liée à la possibilité de témoigner de ces disparus. Parler, nommer les morts, semble ainsi contribuer à les rendre un peu moins absents, un peu moins disparus.

Les qualificatifs employés pour rendre compte de la disparition - " totale ", " complète "- viennent renforcer l'idée d'un anéantissement définitif : la personne se retrouvera dépourvue de tout, même de la possibilité d'exister comme mort auprès des siens. Cette inexistence assoit une forme de continuité entre une vie qui ne vaut pas la peine d'être vécue et une mort inaccessible au deuil. La volonté de devenir " quelqu'un ", de laisser derrière eux une "vie sans rien " est pour la plupart de mes interlocuteurs ce qui motive la migration au risque de la mort.

"On sait qu'on peut laisser la vie, mais on part et on espère réussir bossa une fois, deux fois et on continue, on risque la vie parce que sinon c'est la honte. C'est quand le temps passe et que tu n'as rien, que t'es toujours dans l'aventure ${ }^{26}$, que tu n'as pas d'argent, c'est la honte, mieux vaut mourir. " (Conversation, 03/05/2016)

La " honte " dont parle Moussa Kane, un jeune guinéen, dans l'extrait ci-dessus résume en un mot l'opprobre de l'échec dans la traversée, et plus largement, du projet migratoire, ainsi que l'humiliation d'être considéré, tant par les autres que par soi-même, comme quelqu'un qui n'a pas su avancer, qui reste bloqué en route. S'opposant à la " honte " de ne rien avoir, devenir " quelqu'un" implique la possibilité de sortir de l'invisibilité. C'est cet espoir d'une réinvention de soi que mes interlocuteurs mettent en avant dans leurs discours quand ils expliquent pourquoi ils ont accepté de mettre leur vie en péril. Ils disent tous vouloir échapper à une vie "sans rien ", qu'ils percevaient comme perdue, anonyme ${ }^{27}$. Beaucoup emploient l'expression " partir se chercher en Europe " pour rendre compte de cette volonté de se réaliser. La vie de " morts-vivants" qu'ils rencontrent au cours de leur périple ne fait que renforcer l'acceptation du risque afin de devenir " quelqu'un ". Gaoussou Dembélé décrit ainsi cette vie :

25 Van Gennep (2011) distinguait trois phases dans les rites de passage : la séparation, où l'individu est isolé du groupe, la marge ou liminarité et l'agrégation dans le groupe dans une nouvelle situation sociale. Plus tard, Turner revient sur ces analyses et s'intéresse particulièrement à l'étape intermédiaire du rituel, en insistant sur l'invisibilité structurelle des êtres liminaires (i.e. ils sont structurellement indéfinissables), qui ont une "réalité " physique, mais pas sociale. Plusieurs auteurs reprennent cette notion du rite de passage pour explorer le parcours des migrants (Bayart, 2004 : 418-419 ; Massey et al., 1993 : 452-453; Monsutti, 2007).

26 Bien que la plupart de mes interlocuteurs ne l'utilisaient pas, le terme " aventure " est employé couramment par les migrant·e·s pour décrire leur parcours. Voir à cet égard, les travaux de Pian (2009) et de Bredeloup (2014).

27 Sur ce sentiment d'inexistence, voir les travaux de Timera (2001 et 2009) et de Graw (2012). 
"Depuis que je suis arrivé au Gourougou j'ai compris, le premier jour, j'observais toutes les choses, tout le mouvement, et j'ai compris que le Gourougou c'est le cimetière.

II n'y a pas de vie au Gourougou, tous les gens sont des morts-vivants [silence] parce qu'ils ne sont pas considérés d'abord, ils sont rejetés par la société, ils sont tabassés et maltraités [par les " militaires marocains "], ils ne vivent pas en social, ils sont dans la brousse, dans la forêt, parmi les mauvais esprits. Ils mangent à peine des restes. Seul Dieu les protège [...]. Ils n'ont pas peur de mourir parce qu'ils sont presque morts [...]. Je n'avais pas peur de la mort, à chaque fois que j'ai tenté de frapper la barrière, je me disais que c'était pour être vivant, pour la vie que je tentais ma chance. "

(Entretien, 08/11/2014)

Ce Malien de vingt-huit ans considère que les conditions de vie sur le mont Gourougou, le rejet de la population locale et le traitement de la part des autorités ont fait de lui, après vingt mois au Maroc dont quatorze dans le campement sur la montagne, un " mort-vivant ${ }^{28}$. Sa vie réduite à la survie ressemble à une "vie nue " - qui exprime le simple fait de vivre - et qui s'oppose à la bios, la vie qui vaut la peine d'être vécue (Agamben, 1998). Celle que Gaoussou Dembélé, comme mes autres interlocuteurs, atteindraient en réussissant leurs ambitions au cœur de leur migration.

S'interrogeant sur " what counts as a livable life and a grievable death?", Butler (2006 : 15) distingue également deux formes de vie : les vies qui " comptent " et celles qui " ne comptent pas " et sont sujettes à une forme de précarité et de vulnérabilité perpétuelle, y compris après la mort (qui est invisible et " ungrievable "). Les récits de Mamadou Condé, Cellou Diallo et Ibrahim Sagara renvoient à une forme extrême d'inaccessibilité au deuil en ce qu'il n'est plus seulement question d'effacement public, d'invisibilité sociale, mais aussi - et surtout, selon mes interlocuteurs - d'impossibilité pour les familles de pleurer leurs morts. C'est cette inaccessibilité au deuil très concrète que mes interlocuteurs nomment disparition et qu'ils semblent craindre plus que la mort elle-même. C'est ce que véhiculent les rumeurs que l'on entend depuis des années aux abords de Melilla.

\section{Rumeurs de disparitions, imaginaire de la frontière}

Au printemps 2014, une rumeur circule parmi les migrant·e·s, les bénévoles associatifs et les salariés des institutions de prise en charge des migrant.e.s à Melilla : à chaque tentative de traverser des clôtures, des migrant.e-s meurent et leurs corps disparaissent. Les récits coïncident sur les causes du décès : ces migrant.e.s décèdent dans la confrontation, à la barrière, avec la gendarmerie marocaine et la Guardia Civil. Au cours de mes terrains, j'ai dû entendre une centaine de fois des récits sur les morts à la valla, avec des légères variations selon le locuteur : un jeune ayant fait l'expérience du saut des grillages raconte - avec plus ou moins de détails - que pendant la course frénétique on voit des frères tomber et mourir ; un militant associatif insiste sur l'existence de vidéos où l'on distinguerait des agents des forces de l'ordre agresser et mettre

$28 \mathrm{Si}$ I'on poursuit l'analyse en termes de rite de passage, les conditions de vie décrites par Gaoussou Dembélé renvoient à la mortification caractéristique de la phase liminaire du rituel. 
à mort des migrant.e-s ; un membre d'une ONG qui travaille dans la gestion des migrant.e.s souligne le courage de ceux qui " risquent la mort " à la barrière. À ces différences liées à ce que chacun souhaite mettre en avant - la mort à la barrière, l'existence de possibles preuves inculpant les forces de l'ordre ou le courage des migrant·e.s - s'ajoutent les variations d'intensité dans la façon de rapporter ces histoires. Selon que l'interlocuteur est un riverain, un journaliste, une autorité publique ou une anthropologue, la rumeur prend une forme différente. Mais lorsqu'il s'agit d'expliquer la disparition des corps que personne ne retrouve de part et $d^{\prime}$ autre de la barrière, le récit cède sa place à toute une série d'hypothèses : ils seraient avalés par la terre, mangés par des chiens ou bien enterrés dans des fosses communes ignorées de tous ${ }^{29}$. Un matin de juin 2014, alors qu'on se promène au bord de la plage, Guélor Koumba, un Gabonais de vingt-et-un ans que j'avais rencontré quelques jours auparavant, raconte comment les barrières sont traversées :

" - G. : Tu cours, tu cours, tu fonces sans regarder derrière toi, mais à chaque tentative il y a des morts. Toujours on les voit qu'ils meurent, mais après on ne les retrouve plus [silence]. Ils disparaissent [silence], ils doivent être mangés par des chiens sauvages.

- C. : Mais comment ils meurent?

- G. : Quand tu cours, tu grimpes, tu cours de toutes tes forces, t'es fatigué, fatigué et les Ali ${ }^{30}$ et les Guardia ${ }^{31}$ ils te frappent, coup de pied, bâtons, sur la tête, sur le corps, partout, et tu tombes et ils continuent à te tabasser. C'est comme ça que tu meurs ici.

- C. : Et pourquoi tu dis que les morts disparaissent?

- G. : Ils disparaissent oui. Ils disparaissent. Ils les mettent ailleurs, loin de nous et les bêtes les mangent.

- C. : T'as déjà vu des bêtes manger des gens?

- G. : Non, je n'ai pas vu, mais c'est ce qu'on dit. " (Conversation, 11/06/2014)

La veille de cette discussion, Pedro Ramírez, un avocat membre d'une association des droits de l'homme de Melilla avait employé des termes similaires pour m'expliquer ce qu'il appelait « la réalité de la barrière ":

"II y a beaucoup de morts à la barrière, lors des saltos, ils les voient mourir et après rien, ils disparaissent, ils sont nulle part, personne ne sait ce qui leur arrive [...] et cela sans compter tout ce qui se passe de l'autre côté, là, franchement, on ne sait rien de rien [...]. On peut faire des hypothèses sur le sort de ces corps, des gens disent qu'il y a des fosses communes, mais on n'en sait rien, je ne peux rien te dire de concret sur cette question. " (Entretien, 10/06/2014)

En novembre 2014, les rumeurs courent avec autant d'intensité y compris de I'autre côté de la frontière. À Beni Ensar, ville frontalière marocaine, un jeune guinéen me parle des mystérieuses disparitions des morts :

29 Il ne s'agira pas ici d'évaluer la véracité de ces rumeurs - ce qu'il est toutefois nécessaire et urgent de faire et ce à quoi des associations et des journalistes indépendants consacrent leur énergie et leurs moyens - mais d'explorer leur signification.

30 " Ali " est le terme communément utilisé par les migrant.e.s rencontré·e·s dans la région pour désigner les agents de la gendarmerie royale marocaine.

31 " Guardia " désigne les agents de la Guardia Civil espagnole. 
"On a tout vu, tout entendu ici. À chaque fois qu'on attaque les barrières, il y a des frères qui meurent, mais on ne sait pas ce que deviennent les morts de la barrière. II y a beaucoup de morts, pas à chaque fois, mais il y en a beaucoup quand même. Quand on revient au Gourougou et qu'on fait le compte, on regarde qui est là, qui n'est pas là, on appelle de l'autre côté pour vérifier qui a fait bossa, on constate aussi qu'il y a quelqu'un qui n'est nulle part. On ne sait pas ce qu'ils deviennent, c'est comme si la terre les avalait. " (Conversation, 04/11/2014)

Quel que soit le sort des corps, les hypothèses formulées sur la disparition des morts à la barrière n'ont rien d'apaisant. Contrairement aux analyses classiques qui voyaient dans les rumeurs une manière de réduire les incertitudes (Shibutani, 1966 ; Kapferer, 1989), ces histoires qui circulent aux alentours de Melilla contribuent à façonner un univers quotidien incertain (Bonhomme, 2009 : 154). Ces incertitudes sont associées aux possibilités de mourir et aux probabilités de réussir le franchissement des frontières. Comme toute rumeur, celles-ci sont crédibles au sein du milieu dans lequel elles se déploient (Fine, 2006). Elles révèlent les inquiétudes d'un espace particulier (White, $2000: 83$ ) où il y a une très forte présence des forces de l'ordre. Ces histoires s'appuient sur l'existence avérée d'une gestion brutale des migrant.e.s à la frontière, des abus et mauvais traitements de la part des forces marocaines et espagnoles ${ }^{32}$. Elles mettent en mots la violence des forces de l'ordre ${ }^{33}$.

Plus que de morts, ces rumeurs - qu'elles avancent l'hypothèse de l'action des forces de la nature (corps phagocytés par la terre), des animaux (qui les mangent) ou des hommes (qui les occultent) - parlent de ce que chacun redoute le plus. Ces rumeurs donnent à entendre une dimension particulière du rapport à la mort, qui nourrit les imaginaires - ces horizons flous qui accompagnent l'expérience (Crapanzano, 2004) - de la migration. Les écouter permet de dépasser les récits individuels - comme ceux de Mamadou Condé, Ibrahim Sagara et Cellou Diallo - pour rendre compte de la façon dont la disparition - qui viendrait plonger les migrant·e.s définitivement dans une invisibilité dont ils font déjà en partie l'expérience et dont ils cherchent à tout prix à s'extraire - fait partie de l'imaginaire collectif de la frontière. Ces histoires font de la hantise individuelle une affaire collective voire même une question politique. Car c'est bien la frontière qui est l'agent de la disparition.

Diverses pratiques sont mises en place par les migrant·e.s, individuellement et collectivement, afin d'éviter la disparition.

32 Au-delà des nombreux articles de presse, voir à cet égard le rapport de Human Rights Watch (2014) sur le traitement des migrant.e.s au Maroc de I'année où ces histoires ont été recueillies, ainsi que celui de I'Asociación Pro Derechos Humanos de Andalucía de la même année portant sur les événements survenus à Ceuta en février 2014 lorsque des agents de la Guardia Civil sont accusés d'avoir tiré des balles en caoutchouc sur des migrant·ess qui nageaient dans le but d'atteindre la ville espagnole (APDHA, 2014 : 40-42).

33 Des associations des droits de l'homme et de soutien aux migrant.e.s se sont emparées de cas de mort et de disparition à partir de témoignages de migrants ainsi que de vidéos tournées par des activistes et ont décidé de traduire les agents des forces de l'ordre en justice, portant plainte auprès des tribunaux espagnol et marocain. Malgré leurs recherches, les associations n'ont pour l'instant pas obtenu des informations leur permettant d'attester I'hypothèse de l'enterrement dans des fosses communes, dont parlent beaucoup de personnes gravitant autour du monde lié aux migrant.e.s, contribuant ainsi à faire de "I'autre côté " une zone inconnue, remplie de fantasmes. 
"Je ne veux pas mourir, mais si je meurs j'aurais tenté et je veux que ma famille l'apprenne, qu'ils sachent que j'ai tout donné pour avancer dans la vie. J'ai dit à mon frère [il fait référence à un jeune bissa comme lui, originaire d'un village proche du sien, compagnon de route depuis plus d'un an] que s'il m'arrive quelque chose qu'il appelle, qu'il dise que j'ai fait tout ce que j'ai pu pour ne pas disparaitre complètement. "

(Conversation, 17/04/2016)

Le fait de s'effacer, de ne laisser aucune trace est perçu par Naba Tigré, un Burkinabé de vingt-trois ans, comme tout ce contre quoi il a décidé de s'embarquer dans la migration. Parti pour devenir "quelqu'un ", il redoute de tomber dans un anonymat absolu. Pour l'éviter, il a pris des précautions, il a demandé à un " frère " d'apprendre le numéro de téléphone de sa mère par cœur. Cellou Diallo a lui aussi un "pacte " avec un ami malien qu'il a rencontré au Maroc : " il a le numéro de ma famille au cas où il faudrait annoncer mon décès ". Presque tous mes interlocuteurs avaient conclu ce type d'arrangement avec des compagnons de route afin de prévenir les familles. Nombreux aussi sont ceux qui apportent leur aide dans l'identification des morts, quand un corps sans nom est retrouvé (en faisant circuler des photos, en postant des informations sur Facebook, etc.) Lorsque les autorités marocaines contactaient un prêtre à Nador qui travaillait auprès de migrant.e.s dans la région pour lui annoncer un décès, les différents collectifs de migrant-e-s sur place - regroupés généralement par origine nationale - réussissaient la plupart du temps à apporter des éléments d'identification et à obtenir un contact pour joindre les familles. Dans le même sens, lorsque des personnes étaient absentes à la suite d'une tentative de franchir les clôtures, tout le monde se mobilisait en appelant des pairs des deux côtés de la frontière afin de suivre la trace des disparus, souvent sans succès. C'est que le projet migratoire perd tout son sens si l'anonymat social celui d'une vie vide de sens - se double d'une disparition totale - une mort sans laisser de trace, sans existence.

\section{Conclusion}

De toutes les expériences de la traversée d'Ibrahim Sagara, il y en a une en particulier dont il voudrait faire le récit. II ne s'agit pas d'une histoire vécue directement, mais d'un témoignage qui lui a été rapporté. II voudrait en faire un film parce qu'elle est à ses yeux la plus révélatrice de ce que vivent les migrant·e.s qui veulent gagner l'Europe. II tient cette histoire d'un rescapé d'une embarcation partie de Tanger qui raconte comment devant l'imminence du naufrage les passagers ont commencé à échanger les noms et les numéros de téléphone des proches qu'ils voudraient que les survivants contactent pour annoncer leur décès. Ibrahim - qui est musicien et compose souvent des textes autour de ses expériences de la migration - se veut ici porte-parole. Le récit qu'il a choisi donne à voir et à comprendre ce qui se joue aujourd'hui à la frontière.

L'ensemble de ces discours qui mettent en mots la mort pendant la traversée parle de la violence physique et symbolique des frontières contemporaines. Qu'il s'agisse de récits à la première personne racontant l'expérience du voyage et des morts laissés sur le chemin ou de rumeurs évoquant la disparition des défunts, ces histoires exposent cette violence qui pénètre dans la vie quotidienne des migrant·e.s, qui ponctue leur expérience. Pour les migrant·e.s que j'ai 
rencontré·e·s, la plus grande violence est de ne pas réussir à être "quelqu'un " chez soi et de risquer de n'être plus " personne " en tentant d'échapper à ce destin anonyme ${ }^{34}$. Anonymat auquel contribue la politique d'effacement mise en place par I'Union européenne : absence de protocole commun d'identification des corps retrouvés, inexistence de statistiques officielles des morts aux frontières, silence autour de la question de la responsabilité de ces décès. Si la violence peut ainsi être pensée comme la forme de gouvernement des personnes confinées à des vies à la frontière, la disparition peut être entendue comme l'horizon redoutable qu'instaure la violence du régime contemporain des frontières.

\section{Références bibliographiques}

Agamben Giorgio (1998) Homo Sacer : le pouvoir souverain et la vie nue, Le Seuil, Paris, 213 p.

Agier Michel (2008) Gérer les indésirables. Des camps de réfugiés au gouvernement humanitaire, Paris, Flammarion, $349 \mathrm{p}$.

Albahari Maurizio (2015) Crimes of Peace. Mediterranean Migrations at the World's Deadliest Border, Philadelphia, University of Pennsylvania Press, 288 p.

Andersson Ruben (2014) Illegality Inc. Clandestine Migration and the Business of Bordering Europe, Berkeley, University of California Press, 360 p.

Asociación Pro Derechos Humanos de Andalucía (APDHA) (2016) Derechos humanos en la Frontera Sur, 76 p., [en línea] consultado el 18/08/2016. URL: http://www.apdha.org/media/informe-frontera-sur-2016-web.pdf

Asociación Pro Derechos Humanos de Andalucía (APDHA) (2014) Derechos humanos en la Frontera Sur, 38 p. [en línea] consultado el 14/08/2016. URL: http:// www.apdha.org/media/frontera_sur_2014_web.pdf

Bayart Jean-François (2004) Le gouvernement du monde. Une critique politique de la globalisation, Paris, Fayard, $450 \mathrm{p}$.

Bloch Maurice et Parry Jonathan (1982) Death and the Regeneration of Life, Cambridge, Cambridge University Press, 248 p.

Bondanini Francesco (2014) Migración de Tránsito: Entre temporalidad y largas esperas. El caso del CETI de Melilla, Revista de Antropología Experimental, 14, pp. 189-206.

Bonhomme Julien (2009) Les Voleurs de sexe. Anthropologie d'une rumeur africaine, Paris, Seuil, 208 p.

Bredeloup Sylvie (2014) Migrations d'aventures, Terrains africains, Paris, Éditions du CTHS, $141 \mathrm{p}$.

34 Tous les noms qui apparaissent dans cet article sont fictifs. Bien que cela puisse sembler aller à l'encontre des motivations de mes interlocuteurs, qui se battent contre I'anonymat, il s'agit pour moi avant tout de préserver ceux qui m’ont fait part de dénonciations des pratiques des forces de l'ordre marocaines et espagnoles. Je souhaite, malgré les noms d'emprunt, contribuer à donner de la place à leurs voix et à les rendre visibles comme des acteurs à part entière de leurs trajectoires. 
Butler Judith (2006) Precarious Life. The Powers of Mourning and Violence, London, Verso, $168 \mathrm{p}$.

Clastres Pierre (1974) La société contre l'État, Paris, Éditions de Minuit, pp. 113-136.

Crapanzano Vincent (2004) Imaginative Horizons: An Essay in LiteraryPhilosophical Anthropology, Chicago and London, University of Chicago Press, $260 \mathrm{p}$.

Cuttitta Paolo (2015a) La "frontiérisation » de Lampedusa, comment se construit une frontière, L'Espace Politique, 25 (1), [en ligne] consulté le 29/05/2017. URL : http://journals.openedition.org/espacepolitique/3336

Cuttitta Paolo (2015b) Mare Nostrum e la retorica umanitaria, Intransformazione, Rivista di Storia delle Idee, 4 (1), pp. 128-140.

De León Jason (2015) The Land of Open Graves. Living and Dying on the Migrant Trail, Berkeley, University of California Press, 384 p.

Fassin Didier (2005) Compassion and Repression: The Moral Economy of Immigration Policies in France, Cultural Anthropology, 20 (3), pp. 362-387.

Fine Gary Allan (2006) Rumeur, confiance et société civile. Mémoire collective et cultures de jugement, Diogène, 213 (1), pp. 3-22.

Freud Sigmund (1992 [1905]) Le mot d'esprit et ses rapports avec l'inconscient, Paris, Gallimard, $416 \mathrm{p}$.

Gabrielli Lorenzo (2011) La construction de la politique d'immigration espagnole. Ambiguïtés et ambivalences à travers le cas des migrations ouest-africaines, Thèse de Doctorat en Science Politique, LAM-Sciences Po Bordeaux, [en ligne] consulté le 18/08/2016. URL : https://tel.archives-ouvertes.fr/tel-00599104

Gay-Para Praline (1986) La guerre du rire. Histoires " drôles " au Liban, Cahiers de littérature orale, 20, pp. 103-129.

Graw Knut (2012) On the cause of migration: Being and nothingness in the African-European border zone, in Knut Graw and Samuli Shielke Eds., Global Horizon: Expectations of Migration in Africa and the Middle East, Leuven, Leuven University Press, pp. 23-42.

Heller Charles et Pezzani Lorenzo (2014) Traces liquides : Enquête sur la mort de migrants dans la zone-frontière maritime de l'Union européenne, Revue Européenne des Migrations Internationales, 30 (3-4), pp. 71-107.

Human Rights Watch (2014) Abused and Expelled. Treatment of Sub-Saharan Africans in Morocco, 87 p., [online] last checked on 14/08/2016. URL: https://www. hrw.org/sites/default/files/reports/morocco0214_ForUpload_0.pdf

Kapferer Jean-Noël (1989) Les disparitions de Mourmelon. Origine et interprétation des rumeurs, Revue française de sociologie, 30 (1), pp. 81-89.

Kobelinsky Carolina (2015) Les morts aux frontières de I'Espagne : trajectoire des corps et pratiques locales, in Catherine Withol de Wenden, Camille Schmoll et Hélène Thiollet Éds., Migrations en Méditerranée, Paris, Éditions du CNRS, pp. 1901-201.

Massey Douglas, Arango Joaquín, Hugo Graeme, Kouaouci Ali, Pellegrino Adela and Taylor Edward (1993) Theories of International Migration: A Review and Appraisal, Population and Development Review, 19, pp. 431-466. 
Matard-Bonucci Marie-Anne (1998) Rire sans éclats. Esquisse d'une histoire politique et sociale du rire en régime fasciste, Revue d'histoire moderne et contemporaine, 45 (1), pp. 170-195.

Melly Caroline (2011) Titanic tales of missing men: Reconfigurations of national identity and gendered presence in Dakar, Senegal, American Ethnologist, 38 (2), pp. 361-376.

Migreurop (2012) Atlas des migrants en Europe. Géographie critique des politiques migratoires européennes, Paris, Armand Colin, 144 p.

Monsutti Alessandro (2007) Migration as a Rite of Passage: Young Afghans Building Masculinity and Adulthood in Iran, Iranian Studies, 40 (2), pp. 167-185.

Moussaoui Abderrahmane (2013) Rire en situation de violence. L'Algérie des années 1990, Terrain, 61, pp. 122-133.

Pallister-Wilkins Polly (2015) The Humanitarian Politics of European Border Policing: Frontex and Border Police in Evros, International Political Sociology, $9(1)$, pp. 53-69.

Pian Anaïk (2009) Aux nouvelles frontières de l'Europe. L'aventure incertaine des Sénégalais au Maroc, Paris, La Dispute, 237 p.

Ritaine Évelyne (2015) Blessures de frontière en Méditerranée, Cultures \& Conflits, 99-100, pp. 11-24.

Rodier Claire (2012) Xénophobie business. À quoi servent les contrôles migratoires?, Paris, La Découverte, 200 p.

Schindel Estela (2016) Bare Life at the European Borders. Entanglements of Technology, Society and Nature, Journal of Borderlands Studies, 31 (2), pp. 219-234.

Shibutani Tamotsu (1966) Improvised News. A Sociological Study of Rumor, New York, Bobbs-Merrill, 262 p.

Timera Mahamet (2009) Aventuriers ou orphelins de la migration internationale ? Nouveaux et anciens migrants "subsahariens " au Maroc, Politique africaine, 115, pp. 175-195.

Timera Mahamet (2001) Les migrations des jeunes sahéliens : affirmation de soi et émancipation, Autrepart, 18 (2), pp. 37-49.

Van Gennep Arnold (2011 [1909]) Les rites de passage, Paris, Éditions Picard, $316 \mathrm{p}$.

Weber Leanne and Pickering Sharon (2011) Globalization and Borders: Death at the Global Frontier, Basingstoke and New York, Palgrave Macmillan, 264 p.

White Louise (2000) Speaking with Vampires. Rumor and History in Colonial Africa, Berkeley, University of California Press, 368 p. 


\section{Carolina Kobelinsky}

\section{Exister au risque de disparaître. Récits sur la mort pendant la traversée vers l'Europe}

À Melilla, enclave espagnole bordant la mer Méditerranée, sur le continent africain, de nombreuses histoires sont racontées par les migrant.e.s sur la mort pendant la traversée vers l'Europe. La découverte d'hommes, femmes et enfants noyés, déshydratés, asphyxiés aux frontières est devenue une réalité quotidienne. Contrairement aux frontières maritimes, peu de morts sont comptabilisées et peu de corps sont retrouvés à la barrière de Melilla. La mort est pourtant un spectre qui accompagne l'expérience de toutes les personnes que j'ai rencontrées dans l'enclave et ses alentours. Cette omniprésence spectrale constitue une forme de violence qui s'infiltre tout autant dans les récits des expériences vécues de celles et ceux qui ont réussi à atteindre l'Espagne que dans les rumeurs qui circulent parmi celles et ceux qui attendent encore leur " chance " du côté marocain. L'auteure s'intéresse ici à la forme et au contenu de ces discours, recueillis au cours d'une enquête ethnographique conduite entre 2014 et 2016, en explorant ce qu'ils véhiculent et ce qu'ils disent de la migration aujourd'hui.

\section{Existing at Risk of Disappearing. Narratives on Death during Border Crossing}

In Melilla, Spanish enclave in the Mediterranean, on the African continent, migrants tell numerous stories about death during border crossing. The discovery of men, women and children drowned, dehydrated, asphyxiated at the border has become a daily reality. Unlike maritime borders, few dead are counted and few bodies are found around Melilla. However, death is a specter that accompanies the experience of all the people I met in the enclave and its surroundings. This spectral omnipresence is a form of violence that appears both in stories about lived experiences, told by those who arrived in Spain, and in the rumors that circulate among those who have still been waiting their "chance" on the Moroccan side. The author examine here the form and the content of these narratives, collected during an ethnographic field study carried out between 2014 and 2016, by exploring what they say about contemporary migration.

\section{Existir arriesgando desaparece. \\ Relatos sobre la muerte durante la travesía hacia Europa}

En Melilla, enclave español sobre el Mediterráneo, en el continente africano, los migrantes de paso cuentan numerosas historias sobre la muerte durante la travesía hacia Europa. El descubrimiento de hombres, mujeres y niños ahogados, deshidratados, asfixiados en las fronteras se ha convertido en una realidad cotidiana. Contrariamente a las fronteras marítimas, pocos muertos son contabilizados y pocos cuerpos son encontrados en la frontera de Melilla. La muerte es sin embargo un espectro que acompaña la experiencia de todas las personas que conocí en el enclave y sus alrededores. Esta omnipresencia espectral constituye una forma de violencia que se filtra tanto en los relatos de experiencias vividas por quienes han logrado llegar a España como en los rumores que circulan entre los que todavía esperan tener "suerte» del lado marroquí. En este artículo, la autor examina la forma y el contenido de estos relatos, recolectados durante un trabajo etnográfico llevado a cabo entre 2014 y 2016, explorando lo que vehiculan y lo que dicen de la migración contemporánea. 